\title{
Comparison of Exposure to Risk Factors for Giardiasis Among Endemic and Travel Cases
}

\author{
Alexandra Swirski ${ }^{\star 1}$, David Pearl ${ }^{1}$, Andrew Peregrine ${ }^{1}$ and Katarina Pintar ${ }^{2}$ \\ ${ }^{1}$ University of Guelph, Guelph, ON, Canada; ${ }^{2}$ Public Health Agency of Canada, Ottawa, ON, Canada
}

\section{Objective}

The objective of our study was to determine how demographic and exposure factors related to giardiasis vary between travel (both international and domestic) and endemic cases, for residents of the Region of Waterloo, Ontario.

\section{Introduction}

Increasing immigration to Canada coupled with the increasing number of Canadians travelling domestically and abroad is expected to significantly impact the burden of illness due to enteropathogens, including Giardia, in Canada ${ }^{1}$. When estimating this burden of illness, international travel cases are considered to be distinct from domestically acquired cases due to differences in control measures in other countries ${ }^{1,2}$. However, there is no distinction made between domestic travel-related cases and endemic cases. As such, there has been no published literature where domestic travel-related cases (DTRCs) have been analyzed separately from endemic cases (ECs). This represents a considerable knowledge gap, as risk factors for contracting giardiasis via domestic travel may be different from those associated with endemic giardiasis or international travel. In our study, we subsequently demonstrated that grouping DTRCs and ECs together for analysis is likely not appropriate due to differences in exposures to risk factors for giardiasis among these groups.

\section{Methods}

Public health inspectors gathered exposure and demographic data for giardiasis cases reported in the Region of Waterloo from 2006 to 2012, inclusive. Logistic regression models were fit to assess differences in exposure to risk factors for giardiasis between international travel-related cases and Canadian acquired cases while controlling for age and sex. Multinomial regression models were also fit to assess the differences in risk profiles between international and domestic travel-related cases and endemic cases.

\section{Results}

Over the six year study period, 472 giardiasis cases were reported to the Region of Waterloo, $191(40 \%)$ cases were related to international travel, and $282(60 \%)$ cases were acquired in Canada. Of the cases acquired in Canada, 29 (10\%) were related to domestic travel, and the remaining were acquired within the Region of Waterloo. Travelrelated cases (both international and domestic) were more likely to go camping or kayaking, and consume untreated water compared to endemic cases. Domestic travel-related cases were more likely to visit a petting zoo or farm compared to ECs, and were more likely to swim in freshwater compared to endemic cases and international travelrelated cases. International travelers were more likely to swim in an ocean compared to both DTRCs and ECs.

\section{Conclusions}

Traditionally, domestic travel related cases and endemic cases are grouped together into a domestic category (i.e., Canadian acquired) in studies that examine the effect of travel on the burden of illness on a population. To the best of the authors' knowledge, this is the first study to separate Canadian cases into ECs and DTRCs, instead of the traditional method. We found significant differences in exposures to various risk factors for giardiasis among the various case groups (international travel, domestic travel and endemic), including significant differences between ECs and DTRCs. Therefore, we suggest that in future studies, DTRCs and ECs should not be included together as this may result in missing important associations or risk factors. Distinguishing between these two case types will likely have an impact on public health policies. Perhaps, of greater significance, our findings are important for creating effective and targeted health promotion campaigns to prevent giardiasis in this region, by targeting activity-specific (e.g., endemic, domestic or international travel) risk activities.

\section{Keywords}

giardiasis; travel-related infection; endemic infection; Giardia; surveillance

\section{Acknowledgments}

This research was supported by an infastructure grant to DL Pearl from the Canada Foundation for Innovation and the Ontario Research Fund. The authors would like to thank all of the public health inspectors at the Region of Waterloo Public Health for collecting questionnaire data.

\section{References}

1. Ravel A, Nesbitt A, Marshall B, Sittler N, Pollari F. Description and burden of travel-related cases caused by enteropathogens reported in a Canadian community. J Travel Med. 2010 Jan-Feb; 15(1).

2. Gormley FJ, Rawal N, Little CL. Choose your menu wisely: cuisineassociated food-poisoning risks in restaurants in England and Wales. Epi and Infect. 2012 Jun; 140(6).

\section{${ }^{*}$ Alexandra Swirski}

E-mail: aswirski@uoguelph.ca 